With compliments of the Author 


\title{
Rapid Synthesis of the Ervitsine Alkaloid Skeleton by a Sequential RCM-Heck Cyclization Approach
}

\author{
M.-Lluïsa Bennasar,* Ester Zulaica, Daniel Solé, Sandra Alonso \\ Laboratory of Organic Chemistry, Faculty of Pharmacy, and Institut de Biomedicina (IBUB), University of Barcelona, Av. Joan XXIII, sn, \\ Barcelona 08028, Spain \\ Fax +34(93)4024539; E-mail: bennasar@ub.edu \\ Received 29 October 2007
}

\begin{abstract}
An efficient approach to the bridged framework of the indole alkaloid ervitsine, featuring a ring-closing metathesis reaction from a 2,3-disubstituted indole followed by a vinyl halide Heck cyclization upon the resulting cycloheptene ring, is described.
\end{abstract}

Key words: indoles, annulation, metathesis, Heck reaction, alkaloids

Annulation methodologies involving the indole nucleus are of particular value for synthetic chemists as this heterocyclic moiety represents a common substructure of many biologically active compounds. ${ }^{1}$ Our continuing interest in this area led us to investigate the synthetic possibilities of combining an indole-templated ring-closing metathesis $(\mathrm{RCM})^{2}$ and a vinyl halide Heck cyclization ${ }^{3}$ to rapidly assemble complex bridged structures fused to the indole nucleus, which are present in some indole alkaloids. In this Letter we report the application of this double annulation methodology to the construction of the tetracyclic framework of ervitsine,${ }^{4}$ a unique alkaloid embodying a 2-azabicyclo[4.3.1]decane system fused to the indole ring and two exocyclic alkylidene substituents. ${ }^{5}$
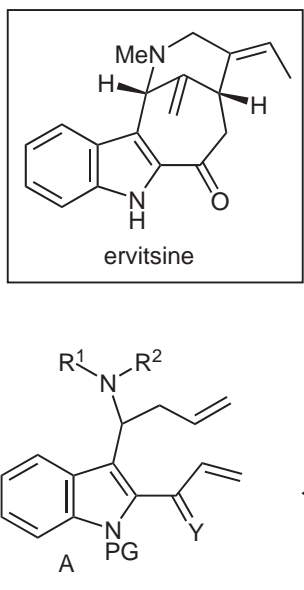

$\mathrm{R}^{1}=\mathrm{Me}$ or $\mathrm{CO}_{2} \mathrm{Me}$

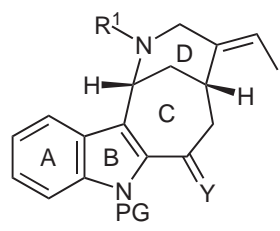

$\Downarrow$ Heck

RCM<smiles>[X]C(=CC)CN([R1])C1CC=CC([Y])c2[nH]c3ccccc3c21</smiles>

$P G$<smiles>[X]C(=CC)CCC</smiles>

Scheme 1 Synthetic strategy

SYNLETT 2008, No. 5, pp 0667-0670

Advanced online publication: 26.02.2008

DOI: 10.1055/s-2008-1032102; Art ID: G39807ST

(c) Georg Thieme Verlag Stuttgart · New York
As shown in Scheme 1, the metathetic ring closure of an indole-containing diene ${ }^{6}$ (A) would provide an indolo 2,3fused cycloheptene ring, with the appropriate functionality for the subsequent intramolecular Heck reaction with the amino-tethered vinyl halide. ${ }^{7,8}$ Similar Heck couplings of vinyl halides and alkenes have proved to be useful for the closure of the piperidine ring in the synthesis of Strychnos alkaloids, ${ }^{9}$ including strychnine ${ }^{10}$ and minfiensine, ${ }^{11}$ as well as in approaches to the geissoschizine ${ }^{12}$ and apogeissoschizine ${ }^{13}$ skeletons.

To establish the feasibility of our proposal for the ervitsine construction, we targeted indolic precursors unfunctionalized at the benzylic $\alpha$-position $(Y=H, H)$, knowing that this methylene group could be eventually oxidized at a later stage of the synthesis. ${ }^{14}$ Protection of the indole nitrogen with a strong electron-withdrawing group was considered critical to guarantee the stability of the gramine [3-(aminomethyl)indole] moiety of the proposed intermediates. Our synthetic route began with the known 2-allyl-3-indolecarbaldehyde $\mathbf{1}^{\text {6c }}$ (Scheme 2), from which an amination-imine allylation sequence was devised to install the homoallylic amine required for the RCM step. Faced with several possibilities, some of them requiring protecting groups, we chose a direct route and incorporated the additional haloalkenyl appendage at the amination step, with the hope that it would be sufficiently inert under the RCM conditions. Thus, reaction of aldehyde 1 with (Z)-2-bromo-2-butenylamine, ${ }^{15}$ followed by alkylation of the resulting imine with allylmagnesium bromide $\left(-78{ }^{\circ} \mathrm{C}\right.$ to r.t.) led to the unstable secondary amine $\mathbf{2 a}$ (not isolated), which was subsequently acylated with methyl chloroformate to give bromo triene $3 \mathbf{a}$ in $60 \%$ overall yield. Similarly, iodo triene $\mathbf{3 b}$ was prepared in $65 \%$ overall yield starting from 1 and (Z)-2-iodo-2-butenylamine. ${ }^{15,16}$

Attention was then directed to the RCM reaction. It was expected that, considering the different substitution and electronic nature of the double bonds of trienes $\mathbf{3}$, the indole-templated cyclization leading to a fused seven-membered ring would be the preferred RCM event. Our expectations were confirmed when $\mathbf{3 a}$ and $\mathbf{3 b}$ on exposure to the second-generation Grubbs catalyst $(\mathrm{Im})\left(\mathrm{PCy}_{3}\right)_{2}(\mathrm{Cl})_{2} \mathrm{Ru}=\mathrm{CHPh}(7 \mathrm{~mol} \%)$ in refluxing $\mathrm{CH}_{2} \mathrm{Cl}_{2}$ gave the desired cyclohepta $[b]$ indoles $\mathbf{4 a}$ and $\mathbf{4 b}$ as the only products in $80 \%$ and $78 \%$ yields, respectively. 
<smiles>C=CCc1c(C=O)c2ccccc2n1S(=O)(=O)c1ccccc1</smiles>

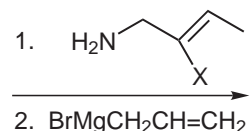

2. $\mathrm{BrMgCH}_{2} \mathrm{CH}=\mathrm{CH}_{2}$

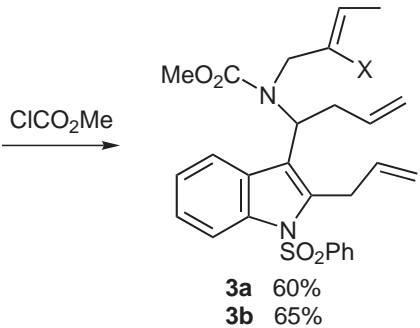<smiles></smiles>

4a $80 \%$

4b $78 \%$

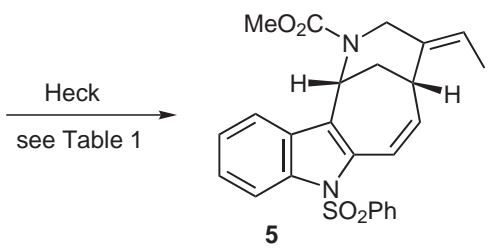

Scheme 2

With a reliable and efficient route to suitably functionalized tricyclic ABC ervitsine substructures, a detailed investigation into the Heck reaction was then performed (Table 1). Our first assays using vinyl bromide $\mathbf{4 a}$ as the substrate were discouraging since under classical polar conditions $^{9 \mathrm{a}}\left[\mathrm{Pd}(\mathrm{OAc})_{2}, \mathrm{Ph}_{3} \mathrm{P}, \mathrm{Et}_{3} \mathrm{~N}, \mathrm{MeCN}\right.$, entry 1] only the starting product was recovered in low yield. On the other hand, the use of ligand-free conditions introduced by Jeffery ${ }^{17}\left[\mathrm{Pd}(\mathrm{OAc})_{2}, \mathrm{~K}_{2} \mathrm{CO}_{3}, \mathrm{TBACl}, \mathrm{DMF}\right.$, entry 2], which had proven successful for the synthesis of related azapolycyclic structures, ${ }^{9 b, 10 a, c, 12}$ resulted in the total decomposition of the material. More satisfactorily, the desired cyclization did proceed upon treatment of $\mathbf{4 a}$ under nonpolar conditions ${ }^{13}$ (palladium catalyst, $\mathrm{Ph}_{3} \mathrm{P}$, proton sponge, $\mathrm{K}_{2} \mathrm{CO}_{3}$, toluene, entries 3 and 4). However, although the conversion yields were good as evidenced by the NMR analysis of the crude reaction mixtures, the isolated yields of the $(E)$-ethylidene tetracycle 5 after column chromatography were only moderate $(30 \%)$, 4 a being invariably recovered even under longer reaction times.

It should be mentioned that the analogous $N$-methyl derivative 7 (Scheme 3), prepared by methylation of the secondary amine $\mathbf{2 a}$ followed by RCM of the resulting tertiary amine $\mathbf{6}$, led to complex reaction mixtures under any of the above Heck conditions. This result seemed to indicate that the presence of a basic nitrogen in the halobutene chain is not compatible with the harsh cyclization conditions, probably due to a competitive dealkylation process. ${ }^{10 \mathrm{c}}$

We proceeded to focus on the more reactive vinyl iodide 4b. When it was subjected to the same nonpolar protocol<smiles>C=CCc1c(C(N)CC=C)c2ccccc2n1S(=O)(=O)c1ccccc1</smiles>

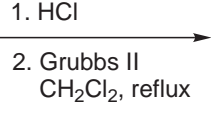<smiles>C/C=C(/Br)CN(C)C1CC=CCc2c1c1ccccc1n2S(=O)(=O)c1ccccc1</smiles>

6 $65 \%$

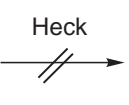

\section{Scheme 3}

(entry 5), tetracycle 5 was obtained only in a slightly better yield $(45 \%)$ along with minor amounts of recovered starting product. Interestingly, we were pleased to find that the addition of $20 \mathrm{~mol} \%$ phenol in combination with $\mathrm{K}_{3} \mathrm{PO}_{4}$ resulted in a cleaner cyclization, giving the ervitsine tetracycle 5 as the only product in $65 \%$ yield (entry $6)$. As far as we know, the use of phenol as a catalytic additive in the Heck reaction is unprecedented, although its positive role in some palladium-catalyzed arylations of ketone enolates has been previously observed by Buchwald. ${ }^{18,19}$ We believe that, according to Buchwald's proposal, ${ }^{18}$ the intermediacy of a palladium phenoxide (e.g. B, Figure 1), which would stabilize an otherwise unstable intermediate, could account for the beneficial effect of the added phenol.

In summary, the RCM-Heck double annulation strategy described here gives short access to the bridged framework of ervitsine from easily accessible indolic precur-

Table 1 Heck Cyclization of Vinyl Halides 4

\begin{tabular}{|c|c|c|c|}
\hline Entry & $\mathrm{X}$ & Reaction conditions & $\begin{array}{l}\text { Products } \\
(\text { yield, } \%)^{\mathrm{a}}\end{array}$ \\
\hline 1 & $\mathrm{Br}$ & $\begin{array}{l}\mathrm{Pd}(\mathrm{OAc})_{2}(16 \%), \mathrm{Ph}_{3} \mathrm{P}(50 \%), \mathrm{Et}_{3} \mathrm{~N}(2 \\
\text { equiv), MeCN, reflux, } 3 \mathrm{~h}\end{array}$ & $\mathbf{4 a}(33)$ \\
\hline 2 & $\mathrm{Br}$ & $\begin{array}{l}\mathrm{Pd}(\mathrm{OAc})_{2}(5 \%), \mathrm{K}_{2} \mathrm{CO}_{3} \text { ( } 5 \text { equiv), } \\
\mathrm{TBACl}\left(1 \text { equiv), DMF, } 60^{\circ} \mathrm{C}, 4 \mathrm{~h}\right.\end{array}$ & - \\
\hline 3 & $\mathrm{Br}$ & $\begin{array}{l}\mathrm{Pd}(\mathrm{OAc})_{2}(5 \%), \mathrm{Ph}_{3} \mathrm{P}(20 \%), \text { proton } \\
\text { sponge }(0.5 \text { equiv }), \mathrm{K}_{2} \mathrm{CO}_{3}(1.1 \text { equiv }) \text {, } \\
\text { toluene, reflux, } 4 \mathrm{~h}\end{array}$ & $\mathbf{5}(30), \mathbf{4 a}(16)$ \\
\hline 4 & $\mathrm{Br}$ & $\begin{array}{l}\mathrm{Pd}\left(\mathrm{Ph}_{3} \mathrm{P}\right)_{4}(5 \%), \text { proton sponge }(0.1 \\
\text { equiv }), \mathrm{K}_{2} \mathrm{CO}_{3}(2.5 \text { equiv }), \text { toluene, } \\
\text { sealed tube, } 2.5 \mathrm{~d}\end{array}$ & $5(30), \mathbf{4 a}(5)$ \\
\hline 5 & $\mathrm{I}$ & $\begin{array}{l}\mathrm{Pd}(\mathrm{OAc})_{2}(10 \%), \mathrm{Ph}_{3} \mathrm{P}(40 \%), \text { proton } \\
\text { sponge }(0.3 \text { equiv }), \mathrm{K}_{2} \mathrm{CO}_{3}(1.5 \text { equiv }) \text {, } \\
\text { toluene, reflux, } 24 \mathrm{~h}\end{array}$ & $\begin{array}{l}\mathbf{5}(45), \mathbf{4 b} \\
(10)\end{array}$ \\
\hline 6 & I & $\begin{array}{l}\mathrm{Pd}\left(\mathrm{Ph}_{3} \mathrm{P}\right)_{4}(10 \%), \mathrm{K}_{3} \mathrm{PO}_{4}(3 \text { equiv }), \mathrm{Et}_{3} \mathrm{~N} \\
(6 \text { equiv }), \mathrm{PhOH}(0.2 \text { equiv }) \text {, toluene, re } \\
\text { flux, } 12 \mathrm{~h}\end{array}$ & \\
\hline
\end{tabular}

${ }^{\text {a }}$ Isolated yields after column chromatography. 


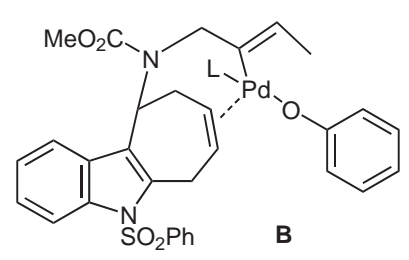

Figure 1 Role of the phenol additive

sors. The application of this approach to closer analogues of this natural product and other polycyclic indole alkaloids is being actively pursued in our laboratory.

\section{Typical Procedure for the RCM Step: Synthesis of Cyclohep- ta $[b]$ indole $4 \mathrm{~b}$}

$(\mathrm{Im})\left(\mathrm{PCy}_{3}\right)(\mathrm{Cl})_{2} \mathrm{Ru}=\mathrm{CHPh}$ (second-generation Grubbs catalyst, 7 mol\%) was added under Ar to a solution of carbamate $3 \mathbf{b}(0.3 \mathrm{~g}, 0.5$ $\mathrm{mmol})$ in $\mathrm{CH}_{2} \mathrm{Cl}_{2}(2.5 \mathrm{~mL})$ and the resulting mixture was heated at reflux for $2.5 \mathrm{~h}$. The reaction mixture was concentrated and the residue was chromatographed $\left(\mathrm{SiO}_{2}\right.$, flash, $96: 4$ hexanes-EtOAc) to give 4b: $0.22 \mathrm{~g}(78 \%)$. ${ }^{1} \mathrm{H} \mathrm{NMR}\left(400 \mathrm{MHz}, \mathrm{CDCl}_{3}\right.$, major rotamer): $\delta=1.51(\mathrm{~d}, J=6.0 \mathrm{~Hz}, 3 \mathrm{H}), 2.59(\mathrm{br}, 1 \mathrm{H}), 2.74(\mathrm{br}, 1 \mathrm{H}), 3.49(\mathrm{~d}$, $J=16.0 \mathrm{~Hz}, 1 \mathrm{H}), 3.71(\mathrm{~d}, J=16.0 \mathrm{~Hz}, 1 \mathrm{H}), 3.78$ (br s, $3 \mathrm{H}), 3.99$ $(\mathrm{m}, 2 \mathrm{H}), 5.25(\mathrm{q}, J=6 \mathrm{~Hz}, 1 \mathrm{H}), 5.72(\mathrm{~m}, 1 \mathrm{H}), 5.86(\mathrm{~m}, 1 \mathrm{H}), 5.95$ $(\mathrm{m}, 1 \mathrm{H}), 7.22(\mathrm{~m}, 1 \mathrm{H}), 7.29(\mathrm{~m}, 2 \mathrm{H}), 7.44(\mathrm{~m}, 2 \mathrm{H}), 7.54(\mathrm{~m}, 1 \mathrm{H})$, $7.71(\mathrm{~m}, 2 \mathrm{H}), 8.23(\mathrm{~d}, J=8.4 \mathrm{~Hz}, 1 \mathrm{H}) .{ }^{13} \mathrm{C} \mathrm{NMR}(74.5 \mathrm{MHz}$, $\left.\mathrm{CDCl}_{3}\right): \delta=21.6\left(\mathrm{CH}_{3}\right), 25.6\left(\mathrm{br}, \mathrm{CH}_{2}\right), 30.9\left(\mathrm{br}, \mathrm{CH}_{2}\right), 52.4(\mathrm{br}$, $\mathrm{CH}), 53.1$ (br, $\mathrm{CH}_{3}$ ), 55.1 (br, $\mathrm{CH}_{2}$ ), 106.9 (br, C), $115.2(\mathrm{CH})$, 118.6 (br, CH), 119.7 (br, C), 124.1 (CH), $124.8(\mathrm{CH}), 126.1(2$ $\mathrm{CH}), 126.4(\mathrm{C}), 128.9(\mathrm{CH}), 129.0(\mathrm{CH}), 129.4(2 \mathrm{CH}), 130.2(\mathrm{CH})$; $133.9(\mathrm{CH}), 136.1$ (C), 137.2 (br, C), 138.8 (C), 156.9 (CO). ESIHRMS $[\mathrm{M}+\mathrm{Na}]^{+}: \mathrm{m} / z$ calcd for $\mathrm{C}_{25} \mathrm{H}_{25} \mathrm{IN}_{2} \mathrm{NaO}_{4} \mathrm{~S}: 599.0472$; found: 599.0474 .

\section{Heck Cyclization of $4 \mathrm{~b}$}

$\mathrm{Pd}\left(\mathrm{Ph}_{3} \mathrm{P}\right)_{4}(17 \mathrm{mg}, 0.015 \mathrm{mmol}), \mathrm{K}_{3} \mathrm{PO}_{4}(96 \mathrm{mg}, 0.45 \mathrm{mmol}), \mathrm{PhOH}$ (3.5 mg, $0.04 \mathrm{mmol})$, and $\mathrm{Et}_{3} \mathrm{~N}(0.1 \mathrm{~mL}, 0.75 \mathrm{mmol})$ were successively added to a solution of vinyl iodide $4 \mathbf{b}(87 \mathrm{mg}, 0.15 \mathrm{mmol})$ in toluene $(11 \mathrm{~mL})$, and the resulting mixture was heated at reflux for $12 \mathrm{~h}$. The reaction mixture was diluted with $\mathrm{Et}_{2} \mathrm{O}$ and washed with a sat. aq $\mathrm{Na}_{2} \mathrm{CO}_{3}$ solution and brine. The organic layer was dried and concentrated. The resulting residue was chromatographed $\left(\mathrm{SiO}_{2}\right.$, flash, hexanes and 5\% hexanes-EtOAc) to give 4- $(E)$-ethylidene-2(methoxycarbonyl)-8-(phenylsulfonyl)-2,3,4,5-tetrahydro-1,5methano- $1 H$-azonino[4,3- $b$ ]indole (5): $43 \mathrm{mg}(65 \%) .{ }^{1} \mathrm{H}$ NMR (400 $\mathrm{MHz}, \mathrm{CDCl}_{3}$, assignment aided by gHSQC, $2: 1$ mixture of rotamers $): \delta=1.70(\mathrm{dm}, J=6.4 \mathrm{~Hz}, 3 \mathrm{H}), 1.91(\mathrm{~d}, J=13.0 \mathrm{~Hz}, 1 \mathrm{H}, 13-$ $\mathrm{H}), 2.25(\mathrm{~m}, 1 \mathrm{H}, 13-\mathrm{H}), 2.94$ and 3.10 (major) $(2 \mathrm{~d}, J=13.5 \mathrm{~Hz}, 1$ $\mathrm{H}, 3-\mathrm{H}), 3.67$ (major) and $3.81\left(2 \mathrm{~s}, 3 \mathrm{H}, \mathrm{OCH}_{3}\right), 3.81$ (masked, $1 \mathrm{H}$, 5-H), 4.04 (major) and $4.17(\mathrm{~d}, J=13.5 \mathrm{~Hz}, 1 \mathrm{H}, 3-\mathrm{H}), 5.37$ (major) and $5.42(2 \mathrm{q}, J=6.4 \mathrm{~Hz}, 1 \mathrm{H}), 5.73$ and 5.91 (major) (2 br s, $1 \mathrm{H}$, 1-H), $6.05(\mathrm{~m}, 1 \mathrm{H}), 7.31(\mathrm{~m}, 1 \mathrm{H}), 7.34(\mathrm{~m}, 1 \mathrm{H}), 7.36(\mathrm{~m}, 2 \mathrm{H}), 7.49$ $(\mathrm{m}, 2 \mathrm{H}), 7.67(\mathrm{~m}, 2 \mathrm{H}), 7.85(\mathrm{~d}, J=8 \mathrm{~Hz}, 1 \mathrm{H}), 8.25(\mathrm{~d}, J=8 \mathrm{~Hz}, 1$ $\mathrm{H}) .{ }^{13} \mathrm{C} \mathrm{NMR}\left(74.5 \mathrm{MHz}, \mathrm{CDCl}_{3}\right.$, assignment aided by gHSQC, major rotamer): $\delta=12.5\left(\mathrm{CH}_{3}\right), 29.3(\mathrm{C}-13), 35.7(\mathrm{C}-5), 45.1(\mathrm{C}-1)$, $45.6(\mathrm{C}-3), 52.7\left(\mathrm{OCH}_{3}\right), 115.7(\mathrm{CH}), 118.6(\mathrm{C}-7), 119.3(\mathrm{C}), 120.2$ $(\mathrm{CH}), 120.3(\mathrm{CH}), 124.5(\mathrm{CH}), 125.6(\mathrm{CH}), 126.2(\mathrm{C}), 126.3(2$ $\mathrm{CH}), 129.1(2 \mathrm{CH}), 133.3(\mathrm{C}), 133.6(\mathrm{CH}), 134.8(\mathrm{C}-6), 136.1(\mathrm{C})$, 136.9 (C), $138.2(\mathrm{C}), 155.1(\mathrm{CO})$. ESI-HRMS $[\mathrm{M}+\mathrm{H}]^{+}: \mathrm{m} / z$ calcd for $\mathrm{C}_{25} \mathrm{H}_{25} \mathrm{~N}_{2} \mathrm{O}_{4} \mathrm{~S}$ : 449.1529; found: 449.1523.

\section{Acknowledgment}

We thank the 'Ministerio de Educación y Ciencia', Spain, for financial support (project CTQ2006-00500/BQU) and the University of Barcelona for a predoctoral grant to $\mathrm{S}$. A.

\section{References}

(1) (a) Sundberg, R. J. Indoles; Academic Press: New York, 1996. (b) Joule, J. A. Science of Synthesis (Houben-Weyl, Methods of Molecular Transformations), Vol. 10; Thieme: Stuttgart, 2000, 361. (c) Somei, M.; Yamada, F. Nat. Prod. Rep. 2007, 24, 843; and previous reviews in this series.

(2) For general reviews, see: (a) Handbook of Metathesis, Vol. 2; Grubbs, R. H., Ed.; Wiley-VCH: Weinheim, 2003. (b) Deiters, A.; Martin, S. F. Chem. Rev. 2004, 104, 2199.

(3) For general reviews, see: (a) Bräse, S.; de Meijere, A. In Metal-Catalyzed Cross-Coupling Reactions; de Meijere, A.; Diederich, F., Eds.; Wiley-WCH: New York, 2004, 217. (b) Zeni, G.; Larock, R. C. Chem. Rev. 2006, 106, 4644.

(4) Joule, J. A. In Indoles, The Monoterpenoid Indole Alkaloids, Vol. 25; Saxton, J. E., Ed.; Wiley: New York, 1983, 232.

(5) For previous synthetic work on ervitsine, see: (a) Bennasar, M.-L.; Vidal, B.; Bosch, J. J. Am. Chem. Soc. 1993, 115, 5340. (b) Bennasar, M.-L.; Vidal, B.; Bosch, J. J. Org. Chem. 1997, 62, 3597. (c) Bennasar, M.-L.; Zulaica, E.; Alonso, Y.; Bosch, J. Tetrahedron: Asymmetry 2003, 14, 469.

(6) For previous work of the authors on RCM reactions of 2,3dialkenylindoles, see: (a) Bennasar, M.-L.; Zulaica, E.; Tummers, S. Tetrahedron Lett. 2004, 45, 6283.

(b) Bennasar, M.-L.; Zulaica, E.; Alonso, S. Tetrahedron Lett. 2005, 46, 7881. (c) Bennasar, M.-L.; Zulaica, E.; Solé, D.; Alonso, S. Tetrahedron 2007, 63, 861.

(7) For the construction of benzo-fused bicyclic systems by sequential RCM and aryl halide Heck reactions, see: (a) Grigg, R.; Sridharan, V.; York, M. Tetrahedron Lett. 1998, 39, 4139. (b) Grigg, R.; York, M. Tetrahedron Lett. 2000, 41, 7255. (c) Lautens, M.; Zunic, V. Can J. Chem. 2004, 82, 399. (d) Enders, D.; Lenzen, A.; Backes, M.; Janeck, C.; Catlin, K.; Lannou, M.-I.; Runsink, J.; Raabe, G. J. Org. Chem. 2005, 70, 10538.

(8) For the construction of indolo-fused bicyclic system by sequential RCM and indolyl halide Heck cyclizations, see: (a) Sunderhaus, J. D.; Dockendorff, C.; Martin, S. F. Org. Lett. 2007, 9, 4223. (b) Ribelin, T. P.; Judd, A. S.; Akritopoulou-Zanze, I.; Henry, R. F.; Cross, J. L.; Whittern, D. N.; Djuric, S. W. Org. Lett. 2007, 9, 5119.

(9) (a) Rawal, V. H.; Michoud, C. Tetrahedron Lett. 1991, 32, 1695. (b) Rawal, V. H.; Michoud, C.; Monestel, R. F. J. Am. Chem. Soc. 1993, 115, 3030.

(10) (a) Rawal, V. H.; Iwasa, S. J. Org. Chem. 1994, 59, 2685. (b) Solé, D.; Bonjoch, J.; García-Rubio, S.; Peidró, E.; Bosch, J. Chem. Eur. J. 2000, 6, 655. (c) Eichberg, M. J.; Dorta, R. L.; Grotjahn, D. B.; Lamottke, K.; Schmidt, M.; Vollhardt, K. P. C. J. Am. Chem. Soc. 2001, 123, 9324.

(11) Dounay, A. B.; Overman, L. E.; Wrobleski, A. D. J. Am. Chem. Soc. 2005, 127, 10186.

(12) Birman, V. B.; Rawal, V. H. Tetrahedron Lett. 1998, 39, 7219 .

(13) Birman, V. B.; Rawal, V. H. J. Org. Chem. 1998, 63, 9146.

(14) For instance, see: (a) Ban, Y.; Yoshida, K.; Goto, J.; Oishi, T.; Takeda, E. Tetrahedron 1983, 39, 3657. (b) Gràcia, J.; Casamitjana, N.; Bonjoch, J.; Bosch, J. J. Org. Chem. 1994, 59, 3939. (c) Saito, M.; Kawamura, M.; Hiroya, K.; Ogasawara, K. J. Chem. Commun. 1997, 765. 
(15) Prepared from (Z)-1,2-dibromo-2-butene or (Z)-1-bromo-2iodo-2-butene.

(16) Solé, D.; Urbaneja, X.; Cordero-Vargas, A.; Bonjoch, J. Tetrahedron 2007, 63, 10177.

(17) (a) Jeffery, T. Tetrahedron Lett. 1985, 26, 2667. (b) Jeffery, T. Tetrahedron 1996, 52, 10113.
(18) Rutherford, J. L.; Rainka, M. P.; Buchwald, S. L. J. Am. Chem. Soc. 2002, 124, 15618.

(19) See also: (a) Solé, D.; Urbaneja, X.; Bonjoch, J. Tetrahedron Lett. 2004, 45, 3131. (b) Solé, D.; Urbaneja, X.; Bonjoch, J. Adv. Synth. Catal. 2004, 346, 1646. 